\title{
Grasping Position Estimation for Prosthetic Hand
}

\author{
Rong Wang, Xiaogang Duan* and Hua Deng \\ State Key Laboratory of High-Performance Complex Manufacturing \\ School of Mechanical \& Electrical Engineering, Central South University, Changsha, 410083, China \\ Corresponding author
}

\begin{abstract}
For multi-freedom prosthetic hand, grasping stability is very important. Force closure method is often used to determine grasping force of each finger. The space position information must be obtained to compute grasping force by using this method. However, for prosthetic hand, the space position is hardly to get because angle sensors cannot be used. Thus dynamics model of tendon-driven prosthetic hand is built and solved by using the finite difference method and newton iteration algorithm base on the information of motor. Numerical simulation demonstrates that the method realizes the goal of calculating position approximately in real time and verifies the high efficiency and enough accuracy of the method.
\end{abstract}

Keywords- stable grasp; fingertip position; dynamics model; numerical simulation

\section{INTRODUCTION}

The problem of position planning of robotic manipulator has been an active area of research in the past years. Robotic dexterous hand or manipulator is designed to finish various complicated tasks, like assembling or precisely operating. Perfect performance requires planning its position and orientation adequately. Simultaneously, control of robotic manipulator to track and grasp a target also needs to know the target's pose and position accurately. Robot dexterous hand or manipulator has made a considerable progress in position planning in recent years. However, the prosthetic hand without encoders or sensors has been scarcely researched.

Vision module or computer vision is usually used to obtain the required information because of its nonintrusive, nondamaging and non-contact advantages [1-5]. Yang et al proposed monocular vision to track the position of object [6]. However, it needs the geometric constraint between target points. Some works [7 8] developed binocular vision to calculate the position of the target based on pictures captured by cameras, and thus realizing automatic position planning using the coordination of target. But its accuracy and robustness are not as good as monocular vision. Shirinzadeh et al used laser interference to realize high precision positioning [9]. However, it is very expensive and has high environment requirement. Encoders have been inseparable parts of robots. Yunong et al planned the joint state in real time via rotary encoders equipped on the manipulator or prosthetic hand and realized the desired trajectory using the encoders or angular sensors to control position and velocity by solving the inverse kinematics [10]. But it does not have high computational efficiency because of the complicated matrix inversion computation.
There is no need for the prosthetic hand to use binocular vision because amputees still have healthy eyes and the equipment is very expensive. Moreover, the method based on sensors is also not applicable for prosthetic hand because it is hard to install it in same case because of the size of hand and the limit of mechanic structure. Consider the particularity of prosthetic hand, under-actuated mechanism is adopted to make the hand anthropomorphic [11]. Prosthetic hand cannot be planned position. The only information can acquired is input voltage of motor which can be used to calculate the input torque of motor. Based on it, this paper attempts to get the angle of joint by solving the forward dynamics equation because the motion of joints are coupled each other, then calculate the position of fingertips through the forward kinematic equation. To solve the non-linear dynamics equation, the high efficiency and accuracy are considered. This paper combines finite difference method (FDM) and newton iterative method to solve the dynamics equation instead of traditional Runge-Kutta algorithm by which the operation is so complex and low-efficient to realize online [12]. And the proposed algorithm can be programmed in matlab and vc software. The numerical simulation verifies the high efficiency and accuracy of this algorithm.

\section{PROBLEM FoRMULATION}

The space position determination methods for tradition robotic manipulator system as shown in Figure 1 are list as follows

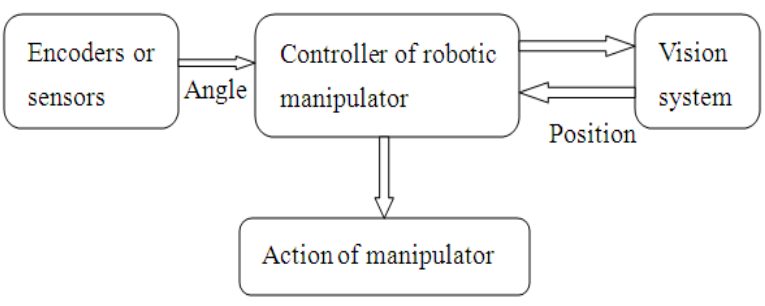

FIGURE I. THE ROBOTIC MANIPULATOR SYSTEM

1) Machine vision module is usually used to get the coordinate position information.

2) Encoders and angle sensors equipped on joints provide the detailed angle information.

For prosthetic hand grasping object stably as shown in Figure 2, space position must be obtained. However, some problems exist as follows 
1) Prosthetic hand is used to help upper limb amputees. So machine vision cannot be adopted.

2) Prosthetic hand with small size and light weight is designed to realize structure and function anthropomorphically. As is shown in Figure 3, the only sensor is equipped on the base joint with the limit of mechanic structure. So the detailed angle information cannot be obtained to calculate the fingertip position.

Thus a grasping position estimation method is presented in the following of paper to achieve it.

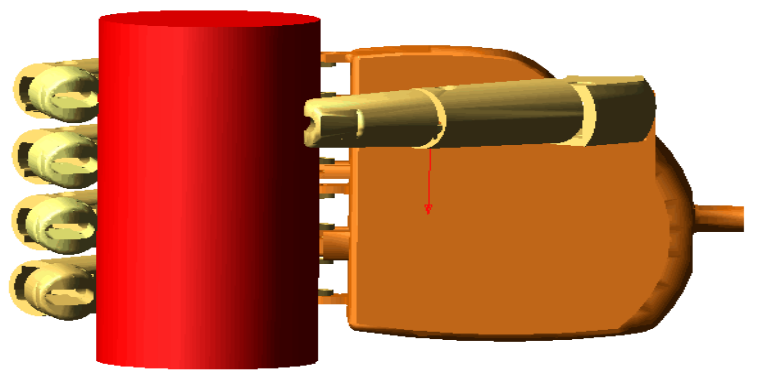

FIGURE II. GRASPING OF PROSTHETIC HAND

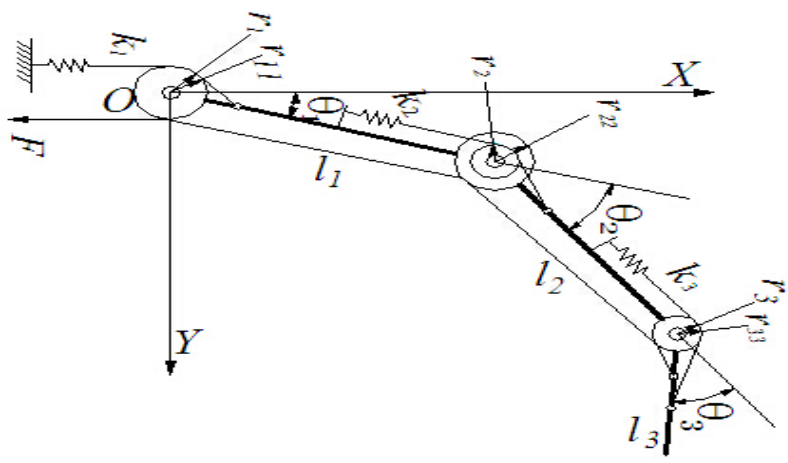

FIGURE III. THE STRUCTURE SKETCH OF PROSTHETIC HAND

\section{Position Calculation of Prosthetic Hand}

\section{A. Solving the Forward Dynamics Equation of Tendor} Actuated Prosthetic Hand

According to the structure of a tendon-driven underactuated prosthetic hand finger, as shown in Figure 3, calculate the potential energy and kinematics energy of system from the point of energy. Using the Lagrange equation method, the dynamics model of prosthetic hand fingers can be established. The formula is shown as follow

$$
M \ddot{q}+H(\dot{q}, q)=\tau_{i}-J F-c \dot{q}-k_{t} q-T_{k}
$$

where $M$ denotes the mass matrix; $H(\dot{q}, q)$ a vector which contains coriolis force and centrifugal force; $\tau_{i}$ denotes the driving toque of each joint; $F$ denotes external force acted on each knuckle, $J$ denotes the mapping matrix from external force acted on each knuckle to corresponding joint; $c \dot{q}$ denotes the speed damping effect items of prosthetic hand; $k_{t} q$ denotes toque of restoration spring, $T_{k}$ denotes pre-toque of restoration spring.

Solving the motion relation between joints of prosthetic hand when the driving toque is given is called the forward dynamics. Equation (1) multiply $M^{-1}$ at both two sides, the angle acceleration motion law of each joint of prosthetic hand is calculated and given by:

$$
\ddot{q}=-M^{-1} H(\dot{q}, q)+M^{-1}\left(\tau-J F-c \dot{q}-k_{t} q-T_{k}\right)
$$

Considering the free grasping period, when grasping in the free space, the external force acted on each knuckle is zero. Supposing there is no spring preload, the dynamics equations becomes

$$
\ddot{q}=-M^{-1} H(\dot{q}, q)+M^{-1}\left(\tau-c \dot{q}-k_{t} q\right)
$$

where $q=q(t)=\left[\begin{array}{lll}\theta_{1}(t) & \theta_{2}(t) & \theta_{3}(t)\end{array}\right]^{T}$ represents the angle displacement of each joint of finger, First use finite difference method to discrete the continuous changing variable, resulting in the form of differential equations, and then solve it. Consider prosthetic hand free grasping period in one second, equally divide the time period with increment $h=0.001$, and express each node with $t_{k}=0.001 k(\mathrm{k}=0,1,2 \ldots)$, thus the corresponding joint angle of each node is $q(k)=\left[\begin{array}{lll}\theta_{1}(k) & \theta_{2}(k) & \theta_{3}(k)\end{array}\right]^{T}$. Assuming the initial value is $q(0)=\left[\begin{array}{lll}0 & 0 & 0\end{array}\right]^{T}$. Instead $\dot{q}$ and $\ddot{q}$ with first-order and second-order differential central difference quotient center differential difference quotient, the detailed form is given as

$$
\dot{q}=\frac{q(k+1)-\mathrm{q}(k-1)}{2 h}
$$

$$
\ddot{q}=\frac{q(k+1)+q(k-1)-2 q(k)}{h^{2}}
$$

Differential equation corresponding to the k-th node dynamics equations has the following form

$$
\left\{\begin{array}{l}
f_{1}\left(\theta_{1}(\mathrm{k}+1), \theta_{2}(\mathrm{k}+1), \theta_{3}(\mathrm{k}+1)\right)=0 \\
f_{2}\left(\theta_{1}(\mathrm{k}+1), \theta_{2}(\mathrm{k}+1), \theta_{3}(\mathrm{k}+1)\right)=0 \\
f_{3}\left(\theta_{1}(\mathrm{k}+1), \theta_{2}(\mathrm{k}+1), \theta_{3}(\mathrm{k}+1)\right)=0
\end{array}\right.
$$

Using the newton iterative method to solve the aforementioned differential equations, and the equation (6) is expressed in a matrix form as follow 


$$
F(X)=0
$$

where

$$
\begin{aligned}
& X=\left[\begin{array}{lll}
x_{1} & x_{2} & x_{3}
\end{array}\right]^{T}=\left[\begin{array}{lll}
\theta_{1}(\mathrm{k}+1) & \theta_{2}(\mathrm{k}+1) & \theta_{3}(\mathrm{k}+1)
\end{array}\right]^{T} \\
& F(\mathrm{X})=\left(\begin{array}{l}
f_{1}(X) \\
f_{2}(X) \\
f_{3}(X)
\end{array}\right)=\left[\begin{array}{l}
f_{1}\left(\theta_{1}(\mathrm{k}+1), \theta_{2}(\mathrm{k}+1), \theta_{3}(\mathrm{k}+1)\right)=0 \\
f_{2}\left(\theta_{1}(\mathrm{k}+1), \theta_{2}(\mathrm{k}+1), \theta_{3}(\mathrm{k}+1)\right)=0 \\
f_{3}\left(\theta_{1}(\mathrm{k}+1), \theta_{2}(\mathrm{k}+1), \theta_{3}(\mathrm{k}+1)\right)=0
\end{array}\right]
\end{aligned}
$$

The Netwon-Raphson iterative formula is given in the following form:

$$
X^{(\mathrm{k}+1)}=X^{(\mathrm{k})}-A\left(X^{(\mathrm{k})}\right)^{-1} F\left(X^{(\mathrm{k})}\right)
$$

where

$$
A\left(X^{(\mathrm{k})}\right)=\frac{\partial F\left(X^{(\mathrm{k})}\right)}{\partial X}=\left[\begin{array}{lll}
\frac{\partial f_{1}\left(X^{(\mathrm{k})}\right)}{\partial x_{1}} & \frac{\partial f_{1}\left(X^{(\mathrm{k})}\right)}{\partial x_{2}} & \frac{\partial f_{1}\left(X^{(\mathrm{k})}\right)}{\partial x_{3}} \\
\frac{\partial f_{2}\left(X^{(\mathrm{k})}\right)}{\partial x_{1}} & \frac{\partial f_{2}\left(X^{(\mathrm{k})}\right)}{\partial x_{2}} & \frac{\partial f_{2}\left(X^{(\mathrm{k})}\right)}{\partial x_{3}} \\
\frac{\partial f_{3}\left(X^{(\mathrm{k})}\right)}{\partial x_{1}} & \frac{\partial f_{3}\left(X^{(\mathrm{k})}\right)}{\partial x_{2}} & \frac{\partial f_{3}\left(X^{(\mathrm{k})}\right)}{\partial x_{3}}
\end{array}\right]
$$

\section{B. Calculation of the Position of Fingertip}

Assume that fingers contact with object at the midpoint of distal phalanges, after solving the inverse dynamics of prosthetic hand, the rotation angle of each joint of fingers are confirmed completely, as shown in Figure 3. According to D-H method and forward kinematics, in the base coordinate system of finger, the position of contact point are given by

$$
\begin{aligned}
& x_{r}=-l_{r, 1} \cos \theta_{r, 1}-l_{r, 2} \cos \left(\theta_{r, 1}+\theta_{r, 2}\right)-l_{r, 3} \cos \left(\theta_{r, 1}+\theta_{r, 2}+\theta_{r, 3}\right) / 2 \\
& y_{r}=l_{r, 1} \sin \theta_{r, 1}+l_{r, 2} \sin \left(\theta_{r, 1}+\theta_{r, 2}\right)+l_{r, 3} \sin \left(\theta_{r, 1}+\theta_{r, 2}+\theta_{r, 3}\right) / 2 \\
& z_{r}=0
\end{aligned}
$$

where $\theta_{r, j}$ and $l_{r, j}(r=1,2, \cdots, R ; j=1,2,3)$ represent the angular displacement and longitude of $r$-th finger and $j$-th finger respectively.

\section{Numerical Simulation OF Prosthetic Hand}

According to the structural parameters of prosthetic hand shown in Table 1, establish the dynamics model first. Using mathematical simulation software Matlab or programming with $\mathrm{C}$ language, the angle displacement can be computed.

The joint angular motion law of prosthetic hand based on finite difference method and Runge-Kutta algorithm are shown as follows
TABLE I. THE STRUCTURAL PARAMETERS OF PROSTHETIC HAND

\begin{tabular}{|c|c|c|c|}
\hline Param & 1 & 2 & 3 \\
\hline$m_{i}(\mathrm{~g})$ & 17.6 & 8.7 & 9.9 \\
\hline$L_{i}(\mathrm{~mm})$ & 55 & 30 & 27 \\
\hline$L c_{i}(\mathrm{~mm})$ & 19.1 & 9.9 & 11.0 \\
\hline$I_{i}\left(\mathrm{~g} \cdot \mathrm{mm}^{2}\right)$ & 1529.4 & 790.8 & 633.4 \\
\hline$k_{i}(\mathrm{~N} / \mathrm{mm})$ & 0.3 & 0.3 & 0.3 \\
\hline$r_{i}(\mathrm{~mm})$ & 5.0 & 3.5 & 2.5 \\
\hline
\end{tabular}

Note: $l_{i}$ denotes the length of i-th link, $m_{i}$ denotes the mass of i-th link, $L c_{i}$ denotes the distance between the center of mass of i-th link and corresponding joint, $r_{i}$ denotes the radius of driving pulley of corresponding joint, $k_{i}$ denotes restoration spring stiffness at the i-th joint, $I_{i}$ denotes rotary inertial of i-th link. In addition, the radius of reel of corresponding joint $r_{21}=5 \mathrm{~mm}$, and damping coefficient $\mathrm{c}=0.3$, the torque of motor is $\tau=\left[\begin{array}{lll}0.2500 & 0.1750 & 0.0875\end{array}\right]^{T}$.

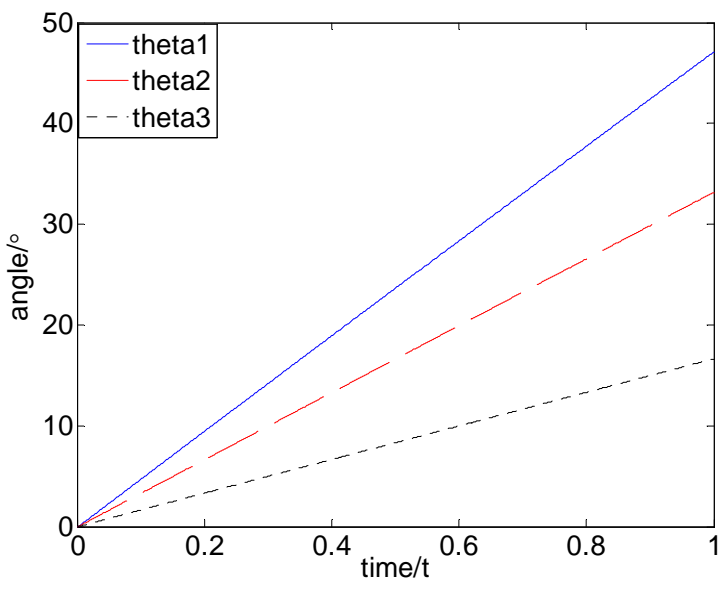

FIGURE IV. SIMULATION RESULT BASED ON FDM

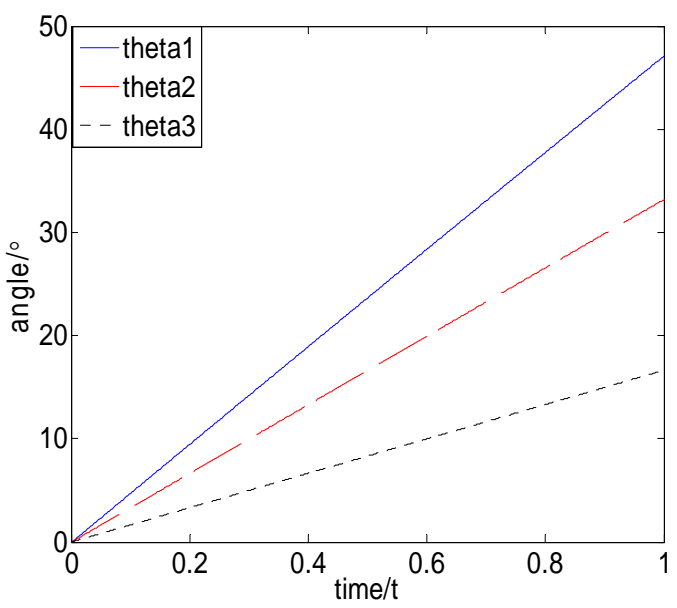

FIGURE V. SIMULATION RESULT BASED ON ODE45 


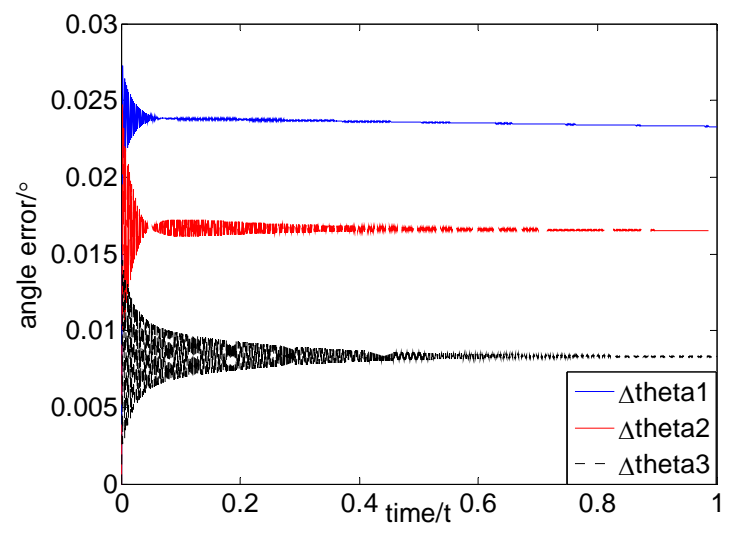

FIGURE VI. THE ERROR OF TWO ALGORITHMS

From the Figure 4 and Figure 5, simulation result based on FDM and Runge-Kutta algorithm are similar, error Figure 6 illustrates that the error is between $0.005^{\circ} \sim 0.025^{\circ}$, the error of proximal joint converges to $0.024^{\circ}$, the error of middle joint converges to $0.017^{\circ}$, and the error of distal joint converges to $0.008^{\circ}$. The select of algorithm have bigger effect on the proximal joint than the other two joint because the motor actuate the proximal joint directly and the torque of other two joints are supplied by the rope and pulley. The error is small totally, so finite difference method have the same high accuracy with Runge-Kutta algorithm and can be used to instead it. Moreover, when simulating in the matlab software, the calculation time Runge-Kutta algorithm spend is about four minutes while the FDM only needs four second. The finite difference method realizes high efficiency which can be used in controller DSP to calculate online and provide reference position before grasping.

\section{CONCLUSIONS}

This paper shows that prosthetic hand can calculate its angle motion law by solving the dynamics equations and calculate the space position approximately, which lay the foundation for stable grasping. The finite difference method (FDM) and newton iterative method are combined to solve the nonlinear and high-order dynamics equation instead of traditional Runge-Kutta algorithm. Numerical simulation demonstrates that our method realizes the goal of calculating position in real time and verifies the high efficiency and enough accuracy of the method..

\section{ACKNOWLEDGMENT}

In this paper, the research was sponsored by the grants from National Basic Research Program 973 of China (Grant No. 2011CB013302).

\section{REFERENCES}

[1] S.S. Mehta, T.F. Burks, Vision-based control of robotic manipulator for citrus harvesting, Comput. Electron. Agric. 102 (2014) 146-158.

[2] B.P. Larouche, Z.H. Zhu, Autonomous robotic capture of noncooperative target using visual servoing and motion predictive control, Auton. Robots 37 (2014) 157-167.
[3] C. Liu, X.H. Huang, M. Wang, Target tracking for visual servoing systems based on an adaptive Kalman filter, Int. J. Adv. Robot. Syst. 9 (2012) 1-12.

[4] S. Hutchinson, G.D. Hager, P.I. Corke, A tutorial on visual servo control, IEEE Trans. Robot. Autom. 12 (1996) 651-670.

[5] D. Kragic, H.I. Christensen, Survey on Visual Servoing for ManipulationComputational Vision and Active Perception Laboratory, Fiskartorpsv.

[6] Yang Y, Cao Q X. Monocular vision based 6D object localization for service robot's intelligent grasping[J]. Computers \& Mathematics with Applications, 2012, 64(5): 1235-1241.

[7] Gorpas D, Politopoulos K, Yova D. A binocular machine vision system for three-dimensional surface measurement of small objects[J]. Computerized Medical Imaging and Graphics, 2007, 31(8): 625-637.

[8] Zhang G, Wei Z, Li X. 3D double-vision inspection based on structured light. ASME J Manuf Sci Eng 2003;125:617-23.

[9] Shirinzadeh B, Teoh P L, Tian Y, et al. Laser interferometry-based guidance methodology for high precision positioning of mechanisms and robots[J]. Robotics and Computer-Integrated Manufacturing, 2010, 26(1): 74-82.

[10] Yunong Zhang, Weibing Li, Xiaotian Yu, Huarong $\mathrm{Wu}$ and Jun Li, Encoder based online motion planning and feedback control of redundant manipulators, Control Engineering Practice,vol.21, no10, 2013,pp.1277-1289.

[11] Yang D, Zhao J, Gu Y, et al. An anthropomorphic robot hand developed based on underactuated mechanism and controlled by EMG signals[J]. Journal of Bionic Engineering, 2009, 6(3): 255-263.

[12] Liu X, Liu M Z. Asymptotic stability of Runge-Kutta methods for nonlinear differential equations with piecewise continuous arguments[J]. Journal of Computational and Applied Mathematics, 2015, 280: 265-274. 